Please cite this paper as follows:

Mousa Marzband, Ebrahim Yousefnejad, Andreas Sumper, José Luis Domínguez-García, Real time experimental implementation of optimum energy management system in standalone Microgrid by using multi-layer ant colony optimization, International Journal of Electrical Power \& Energy Systems, Volume 75, February 2016, Pages 265-274, ISSN 0142-0615, http://dx.doi.org/10.1016/j.ijepes.2015.09.010. 


\title{
Real Time Experimental Implementation of Optimum Energy Management System in Standalone Microgrid by Using Multi-layer Ant Colony Optimization
}

\author{
Mousa Marzband ${ }^{\mathrm{a}, \mathrm{b}}$, Ebrahim Yousefnejad ${ }^{\mathrm{b}}$, Andreas Sumper ${ }^{\mathrm{c}, \mathrm{d}}$, José Luis \\ Domínguez-García ${ }^{c}$ \\ ${ }^{a}$ School of Electrical and Electronic Engineering, Faculty of Engineering and Physical Sciences, Electrical \\ Energy and Power Systems Group, The University of Manchester, Ferranti Building, Manchester, M13 9PL, \\ United kingdom \\ ${ }^{b}$ Department of Electrical Engineering, Lahijan Branch, Islamic Azad University, Lahijan, Guilan, Iran \\ ${ }^{c}$ Catalonia Institute for Energy Research (IREC), Jardins de les Dones de Negre 1, 08930 Sant Adrià de \\ Besòs, Barcelona, Spain \\ ${ }^{d}$ Centre d'Innovació Tecnològica en Convertidors Estàtics i Accionaments (CITCEA-UPC) Departament \\ d'Enginyeria Elèctrica, EU d'Enginyeria TÃĺcnica Industrial de Barcelona, Universitat Politècnica de \\ Catalunya. Barcelona, Spain
}

\begin{abstract}
In this paper, an algorithm for energy management system (EMS) based on multilayer ant colony optimization (EMS-MACO) is presented to find energy scheduling in Microgrid (MG). The aim of study is to figure out the optimum operation of microsources for decreasing the electricity production cost by hourly day-ahead and real time scheduling. The proposed algorithm is based on ant colony optimization (ACO) method and is able to analyze the technical and economic time dependent constraints. This algorithm attempts to meet the required load demand with minimum energy cost in a local energy market (LEM) structure. Performance of MACO is compared with modified conventional EMS (MCEMS) and particle swarm optimization (PSO) based EMS. Analysis of obtained results demonstrates that the system performance is improved also the energy cost is reduced about $20 \%$ and $5 \%$ by applying MACO in comparison with MCEMS and PSO, respectively. Furthermore, the plug and play capability in real time applications is investigated by using different scenarios and the system adequate performance is validated experimentally too.
\end{abstract}

Keywords: Real Time EMS, Short Term Scheduling, Very Short Term Scheduling, Microgrid, Ant Colony Optimization, Optimal Operation.

Email address: mousa.marzband@manchester.ac.uk, Tel. +44(0)1613064654, Fax. +44 (0) 1613064820. Corresponding author (Mousa Marzband) 


\section{Nomenclature}

\section{Acronyms}

\begin{tabular}{|c|c|}
\hline $\mathrm{ACO}$ & ant colony optimization \\
\hline $\mathrm{CCU}$ & central control unit \\
\hline DAM & day-ahead market \\
\hline DER & distributed energy resources \\
\hline DG & distributed generation \\
\hline DR & demand response \\
\hline EGP & excess generated power \\
\hline ES & energy storage \\
\hline EMS & energy management systems \\
\hline EWH & electric water heater \\
\hline FMRTS & five minute real time scheduling \\
\hline HDAS & hourly day ahead scheduling \\
\hline LEM & local energy market \\
\hline MACO & multi-layer ant colony optimization \\
\hline MCP & market clearing price \\
\hline MCEMS & modified conventional EMS \\
\hline MG & microgrid \\
\hline MT & micro-turbine \\
\hline NRL & non-responsive load \\
\hline PSO & particle swarm optimization \\
\hline PV & photovoltaic \\
\hline $\mathrm{ES}+$ & ES during charging mode \\
\hline ES- & ES during discharging mode \\
\hline SOC & state-of-charge \\
\hline TCP & total consumed power \\
\hline UP & undelivered power \\
\hline WT & wind turbine \\
\hline \multicolumn{2}{|c|}{ Variables } \\
\hline \multirow[t]{2}{*}{$\pi^{A}$} & the supply bids by $\mathrm{A}(€ / \mathrm{kWh})$ \\
\hline & $\mathrm{A} \in\{W T, P V, M T, E S-, E S, U P, D R, E G P, \& E W H\}$ \\
\hline$\lambda_{t}^{M C P}$ & $\mathrm{MCP}$ at $t$ in MCEMS $(€ / \mathrm{kWh})$ \\
\hline$\lambda_{t}^{\prime M C P}$ & $\mathrm{MCP}$ at $t$ in EMS-PSO $(€ / \mathrm{kWh})$ \\
\hline$\lambda_{t}^{\prime \prime M C P}$ & $\mathrm{MCP}$ at $t$ in EMS-MACO $(€ / \mathrm{kWh})$ \\
\hline$P_{t}^{A}$ & available power of A in MCEMS (kW) \\
\hline$P_{t}^{\prime A}$ & available power of A in EMS-PSO $(\mathrm{kW})$ \\
\hline$P_{t}^{\prime \prime A}$ & available power of A in EMS-MACO $(\mathrm{kW})$ \\
\hline $\mathrm{A}_{t}^{A}$ & real power set-points of A in MCEMS (kW) \\
\hline $\mathrm{P}_{t}^{\prime A}$ & real power set-points of A in EMS-PSO (kW) \\
\hline $\mathrm{A}_{t}^{\prime \prime A}$ & real power set-points of A in EMS-MACO $(\mathrm{kW})$ \\
\hline$P_{t}^{n}$ & uncontrollable load demand at $\mathrm{t}(\mathrm{kW})$ \\
\hline$S O C_{t}$ & battery SOC in MCEMS (\%) \\
\hline$S O C_{t}^{\prime}$ & battery SOC in EMS-PSO (\%) \\
\hline$S O C_{t}^{\prime \prime}$ & battery SOC in EMS-MACO (\%) \\
\hline$\Delta t$ & time step $(\mathrm{h})$ \\
\hline
\end{tabular}




\section{Introduction}

The increase of distributed generation (DG) penetration in power systems and the introduction of energy markets in recent years have caused numerous challenges in design and planning of power systems based on DG [1]. In the future, consumers would have an isolated MG that includes micro generation systems and their consumption management can be done by EMS according to real time electricity cost.

The main constraints related to renewable energy sources are reliability and dispatchability issues associated with their performance [2]. Since the output power of renewable sources changes with weather conditions, power balance between producers and consumers is considered as a key problem in EMS design. Complex constraints and the impossibility of complete accordance of all DG generation sources with the paradigms of power system has led to the presentation of Microgrid (MG) concept. The main specifications of a MG are as follows:

1. Capability of executing programs such as DR management for controlling the shiftable loads [3];

2. Error tolerance, this tolerance must also be considered for confronting the transient faults [3];

3. Load curtailment ability when the MG cannot feed its load completely or when the electricity prices are high [3];

4. High reliability, power quality, security and system efficiency [3];

5. Self revival which means that the system can revive itself after the occurrence of error in it [3];

6. Plug and play capability of all the devices that are added to the system as microsources with any capacity or are put out of the system is provided automatically by EMS.

For obtaining the characteristics mentioned for MGs, it is necessary to consider short term scheduling (STS) and very short term scheduling (VSTS). Very short and short term economic dispatch are a very important choice in the modern EMS to reduce the operational cost [4-6].

In addition, demand response (DR) is recognized as a very important energy source for cost optimization [3]. Distributed energy resources (DER) significantly increase the number of variables that must enter the economic dispatch problem. STS and VSTS are a large scale, non-convex, nonlinear and time consuming [6]. Therefore, it is necessary to present alternative methodologies for improving the efficiency of these methods against the new paradigms of power system such as heuristic methods [7]. This methodology peresents very fast and adequate response and must be considered for the optimization problems with a lot of variables [8-10].

Ant colony optimization (ACO) algorithm is implemented based on the behavior of real ants that can find the shortest route from the nest to a food source [11, 12]. This method is one of the common methods for optimizing different problems [7]. It presents some advantages in comparison with gravitational search algorithm, artificial bee colony and imperialist competition including usefulness in dynamic applications [13], positive feedback which leads algorithm to rapid discovery of good solutions [14] and distributed computation in order to avoid premature convergence [15]. In this paper, the efficiency of this algorithm in solving problems related to performance optimization, DER scheduling improvement and also the cost reduction of system performance is shown in an MG. This method does not have any special algorithm thus, a proper design should be done. As a result, the algorithm designer has an open hand for increasing its efficiency [16-18]. 
The algorithm presented in this paper has flexibility and adequate fast response to any incident in the system. In the methodology presented, the sources timing schedule with the time intervals day ahead, hour ahead and 5 minutes ahead are considered. STS of energy sources considering intensive penetration of DERs, load curtailment by using DR and plug and play capability are some specific objectives of this paper. The proposed algorithm is implemented and tested experimentally over the IREC's MG system and the experimental results state the proper performance of this algorithm in handling different scenarios occurred in the system. Moreover, the comparison of it with other EMS algorithms shows its better performance.

\section{Problem formulation}

\subsection{The mathematical implementation of EMS optimization problem}

The following assumptions will be considered for the optimization problem within a small MG [7]:

- The voltage level in all of the points of MG is the same;

- The power loss is neglected because of short cabling distance between generation and loads;

- The reactive power flow is neglected.

The optimization problem is defined according to the following objective function:

$$
z \min \mathbb{C}_{\text {Tot }}
$$

where

$$
\mathbb{C}_{T o t} \sum_{t 1}^{m} \mathbb{C}_{t}^{g} \mathbb{C}_{t}^{g} \mathbb{C}_{t}^{E S-}-\mathbb{C}_{t}^{\ell}-\mathbb{C}_{t}^{E S} \Omega_{t} \times \Delta t
$$

where $m$ represent the number of time periods in the scheduling time horizon T; $\mathbb{C}_{t}^{g}$ and $\mathbb{C}_{t}^{g}$ are respectively the cost of energy produced by dispatchable and nondispatchable generation units in period $t ; \mathbb{C}_{t}^{E S}$ and $\mathbb{C}_{t}^{E S-}$ are also cost of energy produced by ES units respectively during charging and discharging operation mode in period $t ; \mathbb{C}_{t}^{\ell}$ state cost of energy consumed by responsive load demand (RLD) and $\Omega_{t}$ justify to depict the penalty cost resulting from undelivered power (UP) during the time period $t$.

The functions related to the costs are obtained as follows

$$
\begin{aligned}
& \mathbb{C}_{t}^{g} \sum_{k 1}^{n^{g}} \pi_{t}^{k, g} \cdot P_{t}^{k, g} \\
& \mathbb{C}_{t}^{\prime g} \sum_{k 1}^{n^{\prime g}} \pi_{t}^{\prime k, g} \cdot P_{t}^{\prime k, g} \\
& \mathbb{C}_{t}^{\ell} \sum_{k 1}^{n^{\ell}} \pi_{t}^{k, \ell} \cdot P_{t}^{k, \ell}
\end{aligned}
$$




$$
\begin{aligned}
& \mathbb{C}_{t}^{E S} \sum_{k 1}^{n^{E S}} \pi_{t}^{k, E S} \cdot X_{t}^{E S} \cdot P_{t}^{k, E S} \\
& \mathbb{C}_{t}^{E S-} \sum_{k 1}^{n^{E S}} \pi_{t}^{k, E S-} \cdot 1-X_{t}^{E S} \cdot P_{t}^{k, E S-} \\
& \Omega_{t} \pi_{t}^{U P} \cdot P_{t}^{U P}
\end{aligned}
$$

where $\pi_{t}^{k, g}$ and $\pi_{t}^{\prime k, g}$ are the offer price by the $\mathrm{k}^{t h}$ dispatchable and nondispatchable electricity generation units during the $t$ time period; $P_{t}^{k, g}$ and $P_{t}^{\prime k, g}$ are defined as the generated power by the $\mathrm{k}^{\text {th }}$; dispatchable and non-dispatchable units during the time period $t ; n^{g}$ and $n^{\prime g}$ are the number of dispatchable and non-dispatchable generation units installed in the MG system; $\pi_{t}^{k, \ell}$ is the offer price by the $\mathrm{k}^{\text {th }}$ RLDs during the time period $t ; P_{t}^{k, \ell}$ is the output power consumed by the $\mathrm{k}^{t h}$ responsive load demands during the time period $t ; \pi_{t}^{U P}$ and $P_{t}^{U P}$ are the amount of UP (the unsatisfied part of non-responsive load- NRL) at time $t$ and its cost, respectively. This term is included in the objective function as a penalty cost for the MG operator to avoid any mismatch in power. $X_{t}^{E S}$ is also defined as a binary variable for ES charge.

The optimization problem has the following constraints:

- Power balance

$$
\begin{aligned}
& \sum_{k 1}^{n^{g}} P_{t}^{k, g} \sum_{k 1}^{n^{\prime g}} P_{t}^{\prime k, g} \sum_{k 1}^{n^{E S}} 1-X_{t}^{E S} \cdot P_{t}^{k, E S-} \\
& P_{t}^{U P} P_{t}^{n} \sum_{k 1}^{n^{\ell}} P_{t}^{k, \ell} \sum_{k 1}^{n^{E S}} X_{t}^{E S} \cdot P_{t}^{k, E S}
\end{aligned}
$$

- Non-dispatchable electricity generation unit boundaries

$$
0 \leq \sum_{k 1}^{n^{g}} P_{t}^{\prime k, g} \leq \bar{P}^{g}
$$

where $\bar{P}^{g}$ is maximum power generated by the non-dispatchable generation units at time $t$.

- Maximum and minimum operating times in the dispatchable generation units [19]

$$
\begin{gathered}
D T_{t-1}^{i}-T^{O N, i} \cdot X_{t-1}^{i}-X_{t}^{i} \geq 0, \forall i, t \\
-D T_{t-1}^{i}-T^{O F F, i} \cdot X_{t}^{i}-X_{t-1}^{i} \geq 0, \forall i, t
\end{gathered}
$$

where $D T_{t}^{i}$ is unit $i$ turn on time period, $T^{O N, i}$ is minimum up-time of unit $i$ (hours), $T^{O F F, i}$ is minimum down-time of unit $i$ (hours), $X_{t}^{i}$ status of unit $i$ at each time $t$ (i.e. 1 when the unit is turn on and 0 otherwise). Also, $P_{t}^{\prime i, g}$ is power generated by unit $i$ at time $t$. 
- Ramp-up and ramp-down limits in the dispatchable generation units

$$
P_{t}^{i, g}-P_{t-1}^{i, g} \leq \bar{R}^{i}
$$

$\bar{R}^{i}$ is maximum generation level of unit $i$.

$$
P_{t-1}^{\prime i, g}-P_{t}^{\prime i, g} \leq \underline{R}^{i}
$$

$\underline{R}^{i}$ is the minimum generation level of unit $i$.

- ES constraints $[7,20,21]$

- Energy storage limits

- Maximum discharge and charge limits

- Energy balance in ES

- Battery SOC

- DR constraints

$$
\begin{gathered}
\sum_{t} P_{t}^{D R} \sum_{t} P_{t}^{U P} \\
P_{t}^{E G P} X_{t}^{E S} \cdot P_{t}^{E S} X_{t}^{D R} \cdot P_{t}^{D R} P_{t}^{E W H} \\
\sum_{t} P_{t}^{E G P} \sum_{t} X_{t}^{E S} \cdot P_{t}^{E S} \sum_{t} P_{t}^{D R} \sum_{t} P_{t}^{E W H}
\end{gathered}
$$

where $X_{t}^{D R}$ is a binary variable for DR status (i.e. 1 if the request is in service and 0 otherwise). Eq. (15) guarantees that the total power consumed by DR should be equal to the total $P_{t}^{U P}$ during daily operation system. Whereas EGP at each interval can be supplied for charging of ES, DR and EWH as formulated in Eq. (16). In addition, the summation of consumed power by these customers should be equal to the summation of EGP during a daily operation system as shown mathematically in Eq. (17).

- Electric water heater (EWH) constraint

$$
P_{t}^{E W H} \leq \bar{P}^{E W H}
$$

In addition to this, for implementing the MACO unit also the $x$ variable is defined with $t$ layers and $i$ allowable values for each layer according to matrix shown in Eq. (19).

$$
\left[\begin{array}{cccccccc}
M P M T_{t}^{M T} & M P W T_{t}^{W T} & M P P V_{t}^{P V} & M P E S d_{t}^{E S-} & M P E S c_{t}^{E S} & M P E W H_{t}^{E W H} & M P D R_{t}^{D R} & M P U P_{t}^{U P} \\
\downarrow & \downarrow & \downarrow & \downarrow & \downarrow & \downarrow & \downarrow & \downarrow \\
P_{1}^{M T, 1} & P_{1}^{W T, 1} & P_{1}^{P V, 1} & P_{1}^{E S-, 1} & P_{1}^{E S, 1} & P_{1}^{E W H, 1} & P_{1}^{D R, 1} & P_{1}^{U P, 1} \\
\vdots & \ddots & \ddots & \ddots & \ddots & \ddots & \ddots & \vdots \\
P_{t}^{M T, i} & P_{t}^{W T, i} & P_{t}^{P V, i} & P_{t}^{E S-, i} & P_{t}^{E S, i} & P_{t}^{E W H, i} & P_{t}^{D R, i} & P_{t}^{U P, i}
\end{array}\right]{ }^{19)}
$$




\subsection{Mathematical implementation of LEM unit}

In this paper, single-sided auction structure is used for finding the value of MCP in each time interval. The required energy by the consumers as well as the energy generated by each of the producers are sent to the LEM unit. This unit is comprehensively explained in the previous papers by the authors $[1,20]$. Therefore, it is not repeated in this paper.

\section{The suggested timing schedule for distributed energy}

The suggested DER scheduling method in this paper consists of optimizing accessible sources with two different time intervals such as hour day ahead and 5 minute ahead. This method is shown in Figure 1.

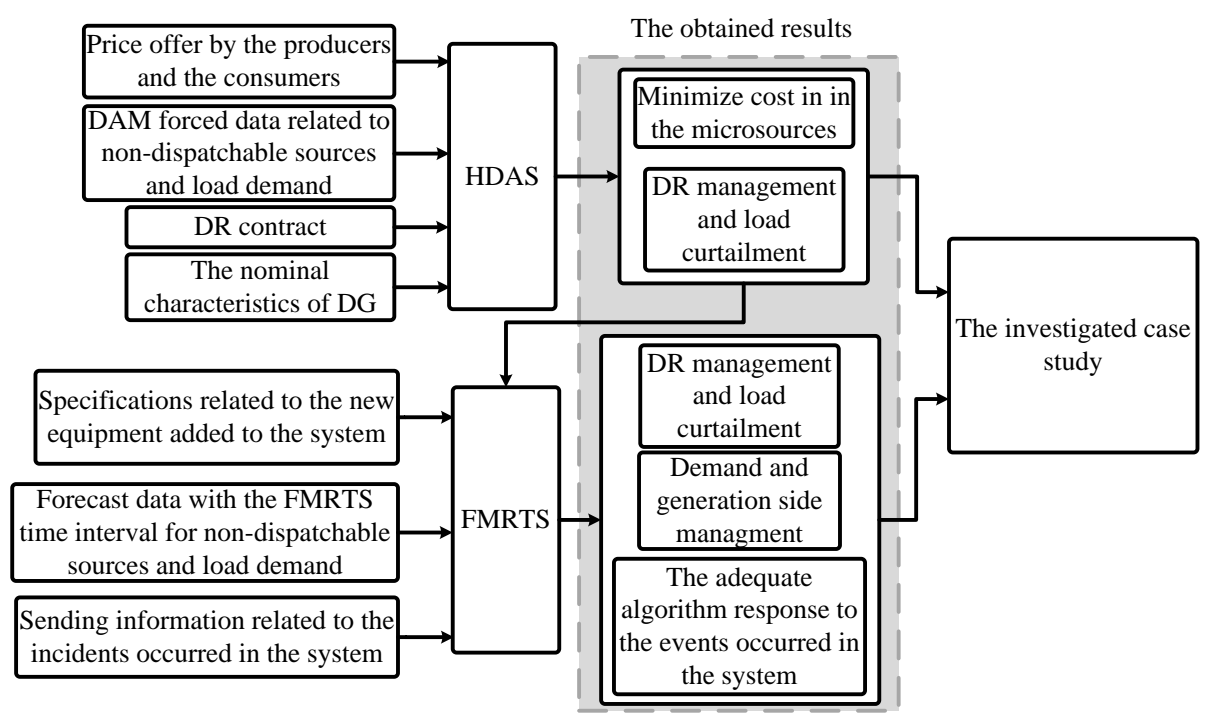

Figure 1: Methodology suggested for investigating HDAS and FMRTS in the isolated system

As it is observed in this figure, this method has hourly day ahead scheduling (HDAS) and five minute real time scheduling (FMRTS) blocks. Day ahead scheduling is used as input data for the HDAS method. It is also seen in the figure that the inputs related to HDAS block are energy price offers, the contract related to DR and the specifications related to DER sources considered in the system. In FMRTS block various inputs are considered including the information related to forecast data for both non-dispatchable energy sources and loads, the specification of equipment connection/disconnection to the system, information related to the incidents and also execution time of those.

The information received by the central control unit (CCU) from the HDAS block includes load management and curtailment signals and DR value with minimum operation cost.

The FMRTS block output has the responsibility of load and DR management and all real time scheduling. In real time scheduling the aim of CCU is to find the best and fastest method for responding to the incident occurred in the system and to give DERs and consumers the necessary orders. 


\section{The proposed Algorithm for EMS}

In this paper, three different algorithms for implementing EMS based on LEM by using heuristic techniques and no optimization method are presented as shown in Figure 2. MCEMS and EMS-PSO mathematical implementation are explained in $[1,7]$ in detail and therefore not treated in this paper. Hence, only EMS-MACO algorithm is described. This algorithm is composed of two units. The performance of EMS unit is explained in the following subsection.

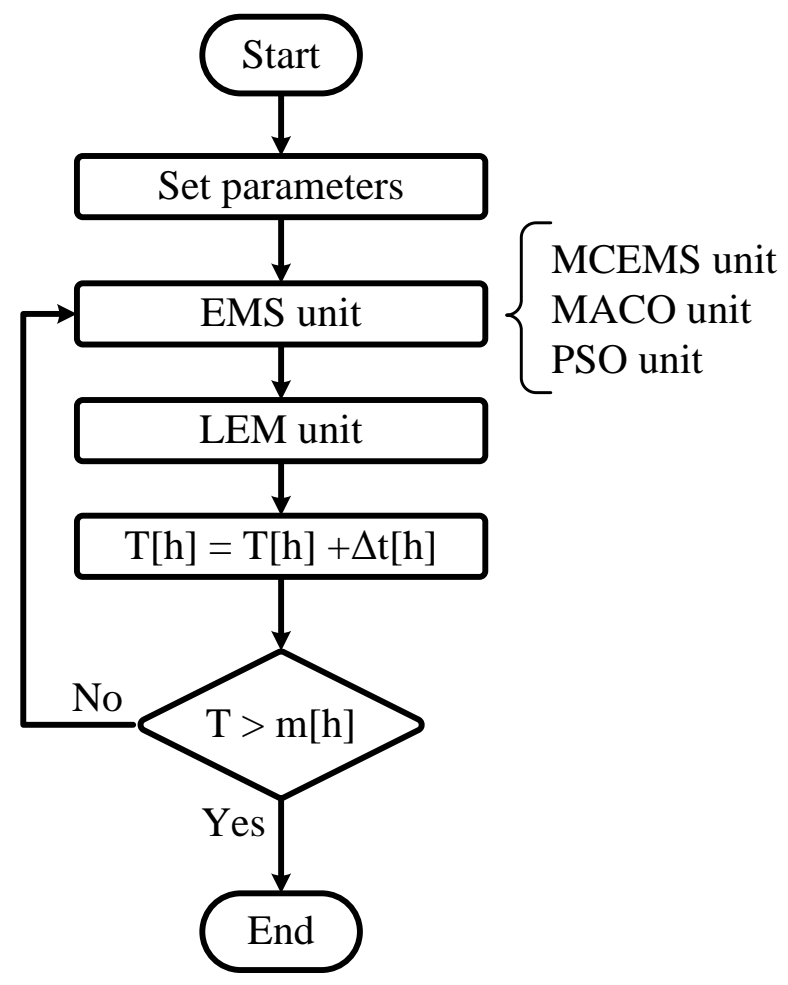

Figure 2: The proposed algorithms for EMS

\subsection{EMS-MACO algorithm}

\subsubsection{MACO unit}

ACO uses the performance of the ants in stepwise improving of the movement path. Eventually, they can help each other to find the shortest path from the nest to a food source. This method is such that each of the ants first randomly chooses a way for reaching food and returning home. Along the path they leave a substance from themselves called pheromone that in the next passes helps them in finding it. The concentration of this substance increases/decreases with the increment/decrement of the ants passing. The shorter the pass, the shorter the time spent for one time going and returning. As a result in a certain time interval the number of goings and returns increases [11, 12, 17, 18].

Finally all the ants will use a route which is the shortest one. This algorithm can be used efficiently in different optimization problems. In the optimization process of the system cost function by the ants algorithm, the powers generated by the microsources 
$\left(P_{t}^{M T}, P_{t}^{W T}, P_{t}^{P V}, P_{t}^{E S-}\right)$ and the power consumed by the consumers $\left(P_{t}^{M T}, P_{t}^{E W H}\right)$ and $P_{t}^{D R}$ are dependent on each other. This approach is based on demand side management considering physical relations and constraints presented in Section 2.1. The previous and next energy stored in the battery during charging and discharging mode is very important. So, there is no possibility for random selection of the value for each of charging and discharging powers separately at every iteration. The process of executing MACO optimization algorithm is shown in Figure 3, in which the number of layers is equal to the number of design variables and the number of nodes in each particular layer is equal to the number of allowable values corresponding to each variable. As it is observed in this figure, the problem of cost function optimization is considered with 48 layers (variables) and $i$ allowable values according to technical constraints for each layer. Each allowable value is defined by a set including these powers, and by considering physical relations and constraints presented in Section 2.1. By selecting the suitable cost function and using the algorithm of Figure 3, allowable values in each layer will be obtained with the minimum Cost of Energy. These ants randomly choose the allowable values and in each time interval this process will be repeated from 00:00 to $23: 30$. The probability of selecting each of the allowable values in the first iteration is considered equal by placing the same pheromone over them. After all, the ants reach the time 23:30, cost function is calculated for each ant and the least cost function is selected. The pheromone of the route with the least expense is increased; as a result, this attempt will raise the possibility of choosing this route by the ants in the next iteration. For this reason, the pheromone of other routes is decreased. In this way, again the ants randomly start choosing the allowable values and routes while this time the chance of selecting values that are in the optimum path is higher. By repeating this process, the algorithm converges and finally a path will be obtained that all the ants will pass it in which this route provides the minimum cost function. The proposed unit is illustrated by a Pseudo-code in Algorithm 1.

\section{Application to test grid}

Simulation and experimental evaluations are performed for a stand-alone wind turbine (WT)/ Photovoltaic (PV)/ Microturbine (MT) and ES system. IREC Testbed is shown in Figure 4. The shown converters are capable to emulate any type of generation and consumption. Detailed explanation concerning the structure and applying configuration setting are presented in [1]. As observed in Figure 4, this system has a central controller which some data will be sent to it. The data includes offer of each of the producers, the value of predicted power related to non-dispatchable sources and load, the value of stored energy in the battery in the previous time and the general properties of each micro-source (such as maximum and minimum power generated by them, the turning on and turning off time of the microturbines). Then, the controller can make required decisions for exchanging energy between micro-sources and the amount of consumed power by applying the algorithm presented for EMS.

The real life experimental data extracted from [1] are also used to emulate WT, PV and NRL. The used wind data is obtained from the weather station at Museu de Badalona, Badalona (Spain) affiliated with the Generalitat de Catalunya Weather Network. The hourly average wind speed data recorded at a height of 20 meters was chosen for the 24-hour simulation study [22] and measured from the wind turbine existing at the IREC's roof. The solar data is obtained from the online records of the Manresa, Barcelona (Spain) [23]. In Figure 5, WT and PV profiles are shown for different scenarios considered. As it is observed in Figure 5, WT has a defect during the evening 


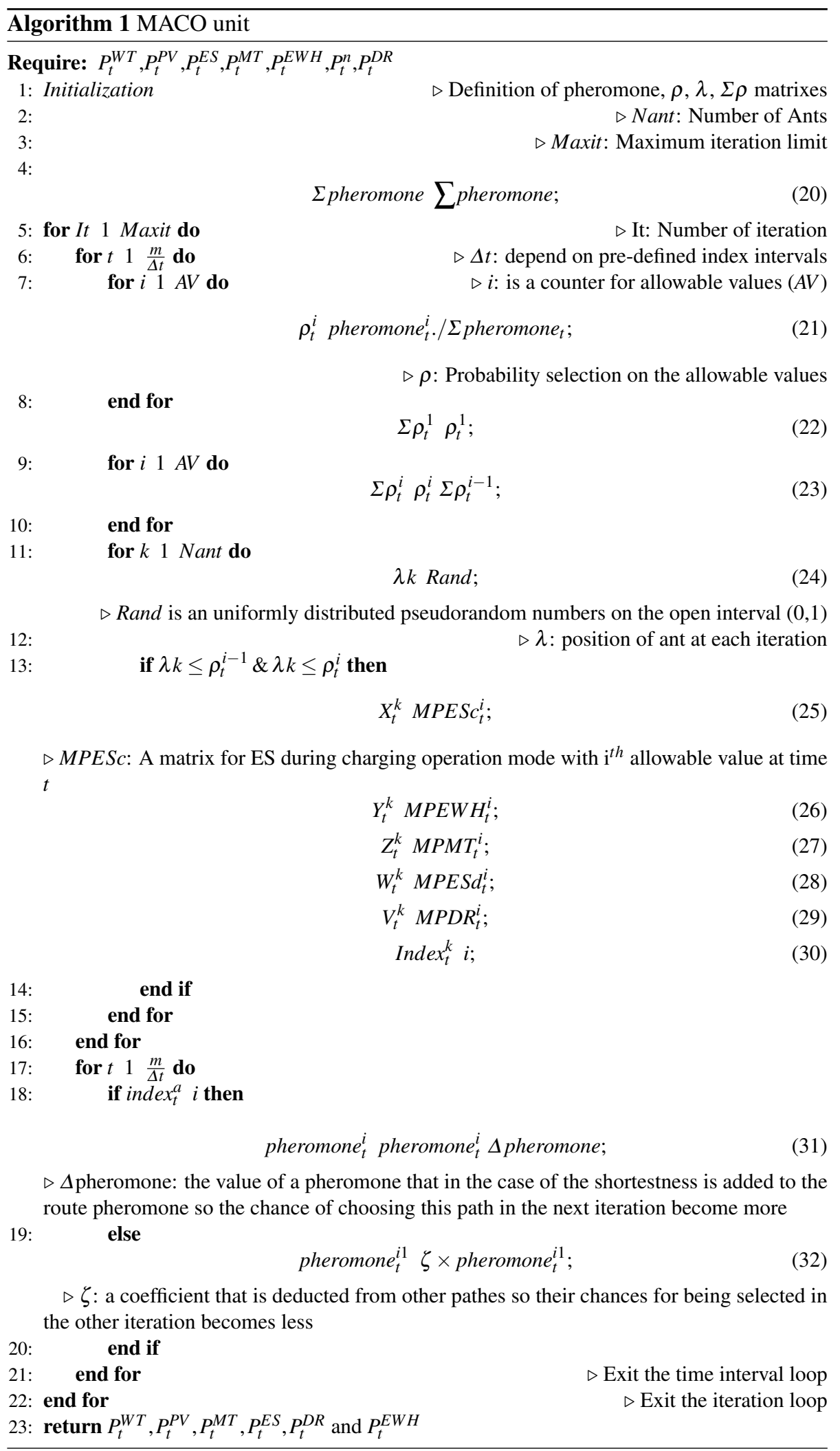




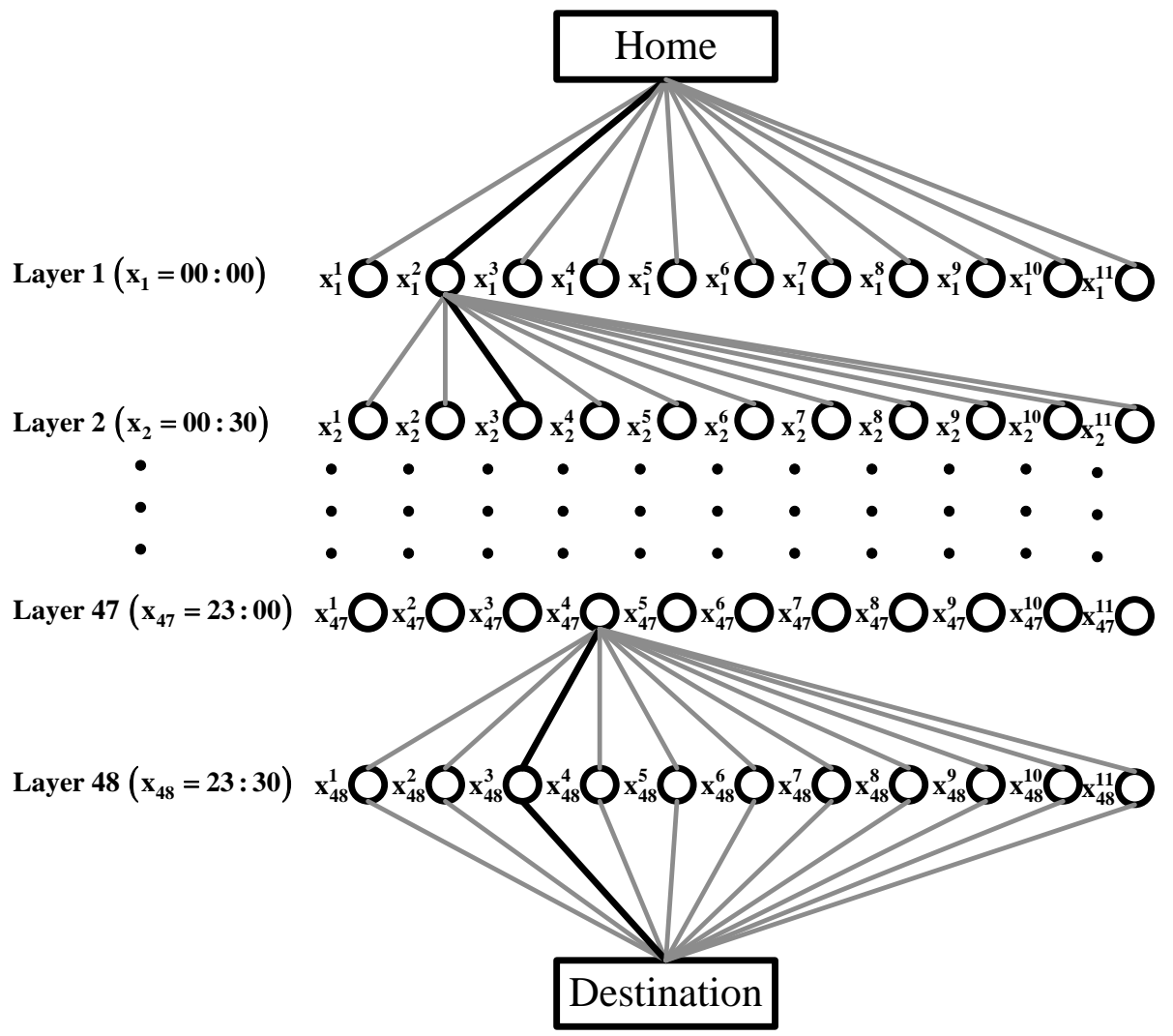

Figure 3: Graphical representation of the ACO process in the form of a multi-layered network

and is put out of service. Also, PV has gone out of service because there is no longer sun.

Load demand profile considered is an average load profile of Spain obtained from [25] which is shown in Figure 6. Load demand increases due to peaks of consumption.

Three scenarios are considered to evaluate the performance and accuracy of the proposed algorithm.

- scenario $\sharp 1$ : Normal operation

- scenario $\sharp 2$ : Sudden load increase

- scenario $\sharp 3$ : Plug and play ability

The suggested EMS algorithms shall have the ability of finding the best solution to fulfill load demand also load curtailment considering optimal scheduling and operation of DERs and ES units. As a result, the proposed algorithms are able to manage DR during system daily performance. In TABLE 1 , the constant offer prices used for different devices are reported. 


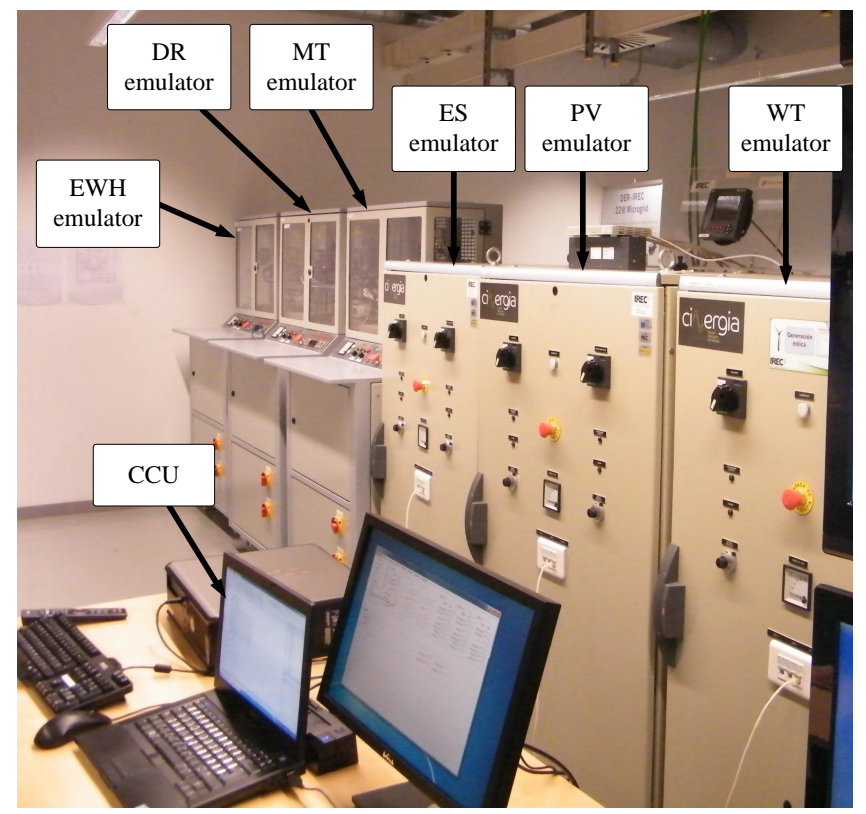

(a) IREC's MG

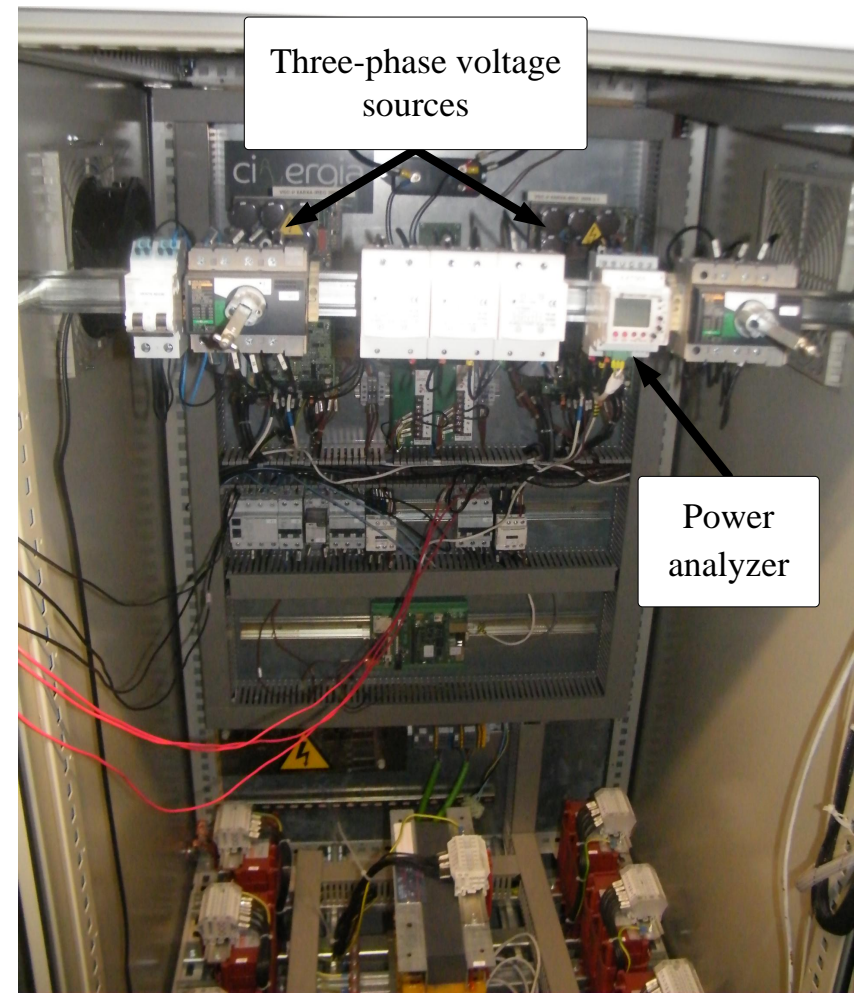

(b) Cabinet inside details

Figure 4: System configuration of IREC's MG Testbed 


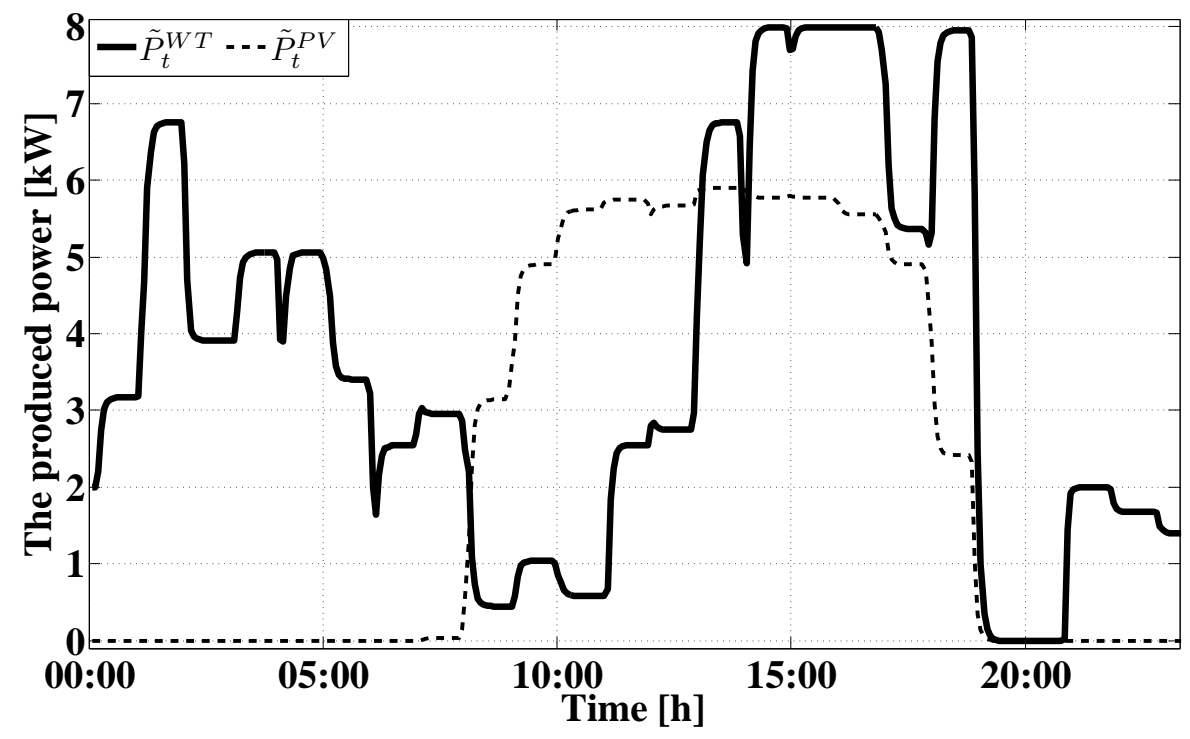

Figure 5: WT and PV emulator profiles during the system daily operation [23, 24]

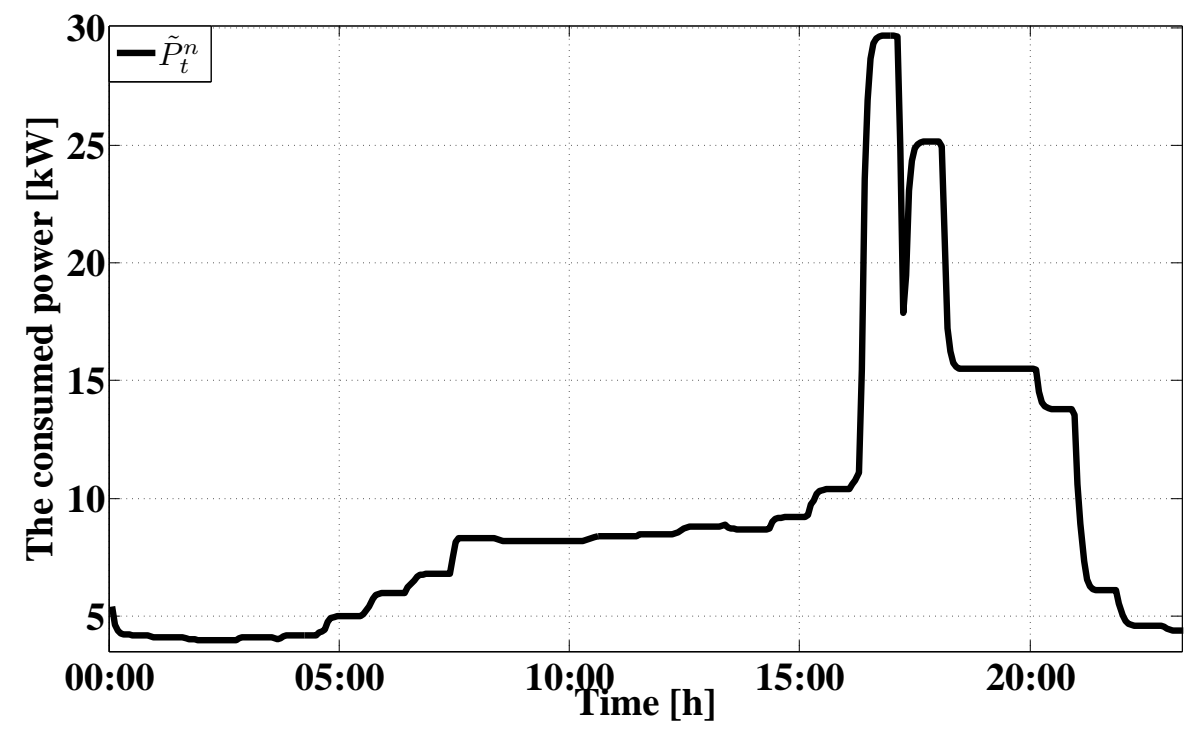

Figure 6: Load emulator profile during the system daily operation [25]

\section{Results and discussion}

This section presents the comparative results of some experimental evaluation of MCEMS, PSO and MACO in EMS application over the islanded IREC's MG. These experiments are carried out to verify the EMS operation under different scenarios.

ES and SOC profiles for each of the three presented algorithms are shown in Figs. 7(a) and 7(b). The initial value of SOC is considered to be $50 \%$ for all of the algorithms. As it is observed in Figure 7(b), the ES system is almost operated in a sim- 
Table 1: The supply bids by generation units into a supply curve [€/kWh]

\begin{tabular}{llllllll}
\hline \hline$\pi^{W T}$ & $\pi^{P V}$ & $\pi^{M T}$ & $\pi^{E S-}$ & $\pi^{E S}$ & $\pi^{U P}$ & $\pi^{D R}$ & $\pi^{E W H}$ \\
\hline 0.083 & 0.112 & 0.17 & 0.112 & 0.083 & 1.5 & 0.115 & 0.105 \\
\hline
\end{tabular}

Table 2: Run time and total generation cost for case study corresponding to 100 iteration of the heuristic EMS algorithms

\begin{tabular}{lll}
\hline \hline & MACO & PSO \\
\hline Execution time (S) & 1.14 & 27.45 \\
Total generation cost $(€)$ & 35.35 & 36.42 \\
Error $(\%)$ & 1.26 & 4.32 \\
\hline
\end{tabular}

ilar operating mode in both EMS-MACO and EMS-PSO algorithms. ES is operated in charging mode in about $40 \%$ of the day based on the optimization algorithm. Although, MT offer price is higher than ES, EMS decided to use MT for a longer time. SOC values in those algorithms is approximately equal during 06:00-12:00. But it is noteworthy that in optimization algorithms, the value is above $55 \%$ for $58 \%$ of the time. This fact shows that optimization algorithms are storing more energy than MCEMS which can be used in the case of fault. Moreover, the less usage of ES results in the higher reliability of the energy storage, and the system under any condition. At the end of the day, SOC level is $24 \%$ and $69 \%$, for MCEMS and the optimization algorithms, respectively which implies better backup plan to ensure availability and reliability of the system for the next day in case of a power outage. Eventually, it is remarkable that although the response of both optimization algorithms (PSO and MACO) are similar, MACO reduces the stress on the ES with slower changing periods compared to PSO.

In Figure 8, the bar graphs related to the ES power during charging and discharging, EWH, UP and excess generated power (EGP) are shown. As it is observed in Figure 8(a) (MCEMS case), in most of the time, ES is operated in charging mode. This charging period is mainly concentrated in the time range of 10:00 to 17:00. During the rest of the period, ES is usually discharging. Another point about this figure is that EWH is also connected during this period; thus, there exist low power demand and large power generation. Finally, as it is seen in Figure 8(a), there is a period in the evening which there exist some UP there.

Note that the average cost obtained by the EMS-MACO is the lowest compared with MCEMS and PSO cases. In other words, optimization techniques provide lower costs comparing to conventional EMS. Although, based on Table 3, the values in PSO is close to MCEMS values in several periods of the day, there are some differences during the first period of the day (due to the high selling price of the ES is noticable). Finally, note that at the last time period the cost is higher because of the penalty function affected by the UP.

The value of MCP is shown in Figure 9 in each time interval. Its average value is also mentioned in each 6 hours period in Table 3. As it is observed in the figure, in all the time intervals (except 19:00) the value of $\lambda_{t}^{M C P}$ is greater than $\lambda_{t}^{\prime M C P}$. Maximum difference between $\lambda_{t}^{M C P}$ and $\lambda_{t}^{I M C P}$ is equal to $0.44 € / \mathrm{kWh}$ that occurs at 08:00 


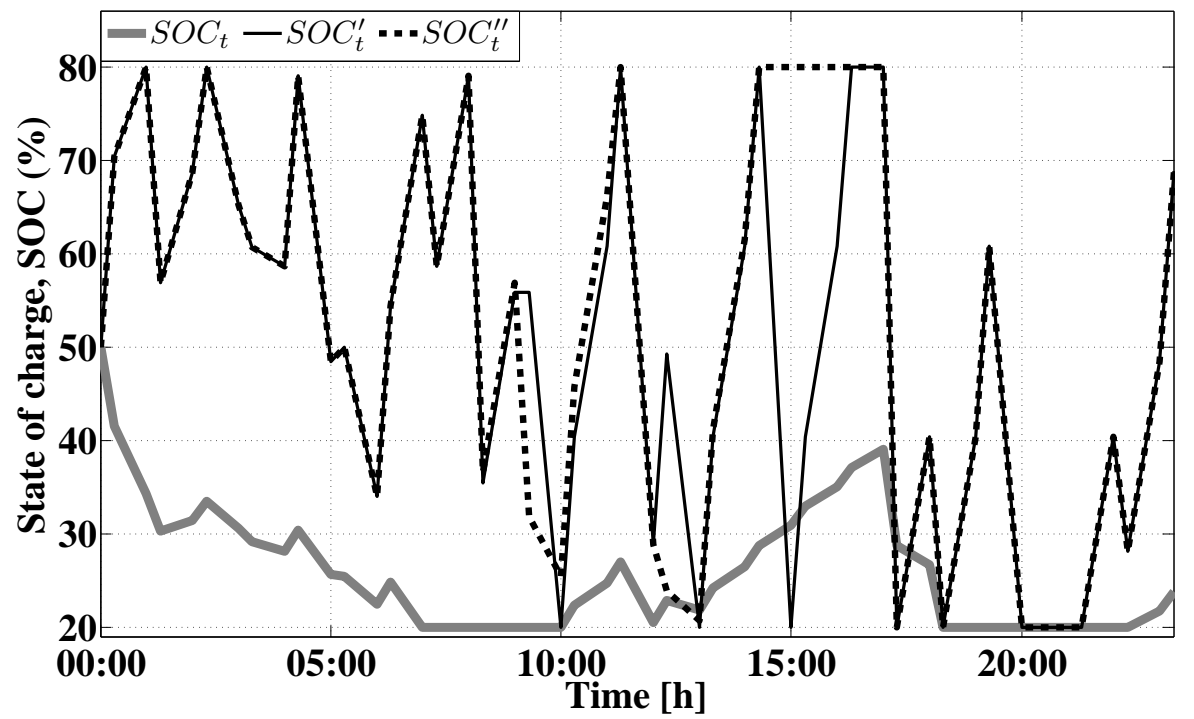

(a) SOC during system daily operation

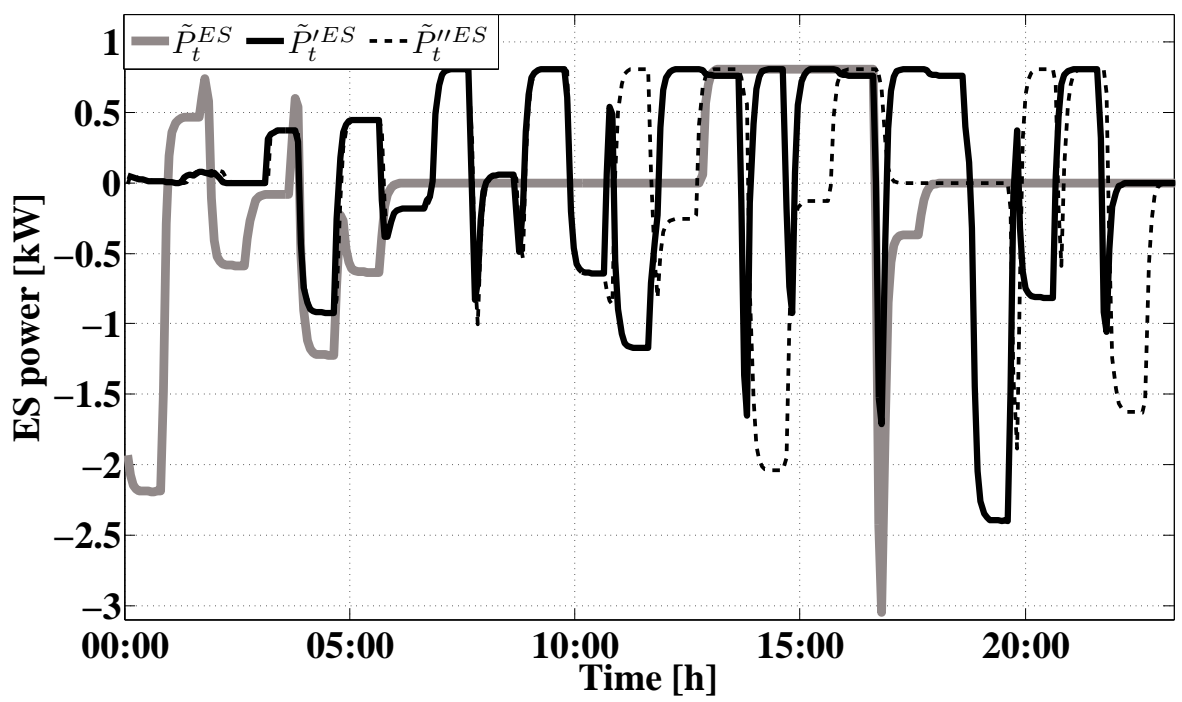

(b) ES power (Solid light-gray line indicates MCEMS algorithm. Also, solid and dash black lines represent output of EMS-PSO and EMS-MACO algorithms, respectively

Figure 7: ES profile during the system daily operation

$\mathrm{o}^{\prime}$ clock. In this hour, the values of $P_{t}^{T C P}$ is equal to $P_{t}^{\prime T C P}$. This fact shows that at this hour the amount of money that must be spent on total consumed power (TCP) in the optimization algorithms is much less than the value of $\lambda_{t}^{\prime M C P}$. At the rest of the hours their values are equal. Maximum value of $\lambda_{t}^{M C P}$ is equal to $1.32 € / \mathrm{kWh}$ that is at $18: 00$ 18 :30 when scenario $\sharp 2$ has occurred. Maximum value for $\lambda_{t}^{\prime M C P}$ is obtained at hours that scenarios $\sharp 2$ and $\sharp 3$ have occurred. Also, the minimum value of $\lambda_{t}^{M C P}$ is obtained at the intervals 03:30-04:00 and 05:30-06:00. The minimum value for $\lambda_{t}^{\prime M C P}$ is equal 


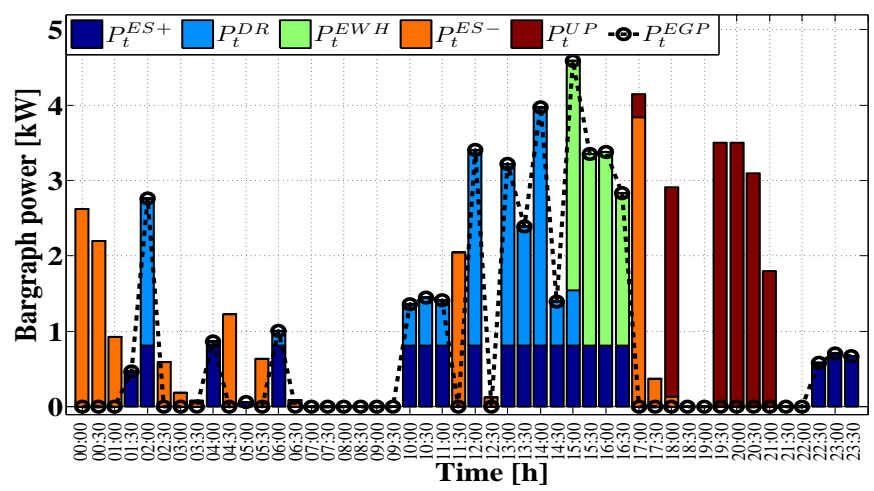

(a) MCEMS

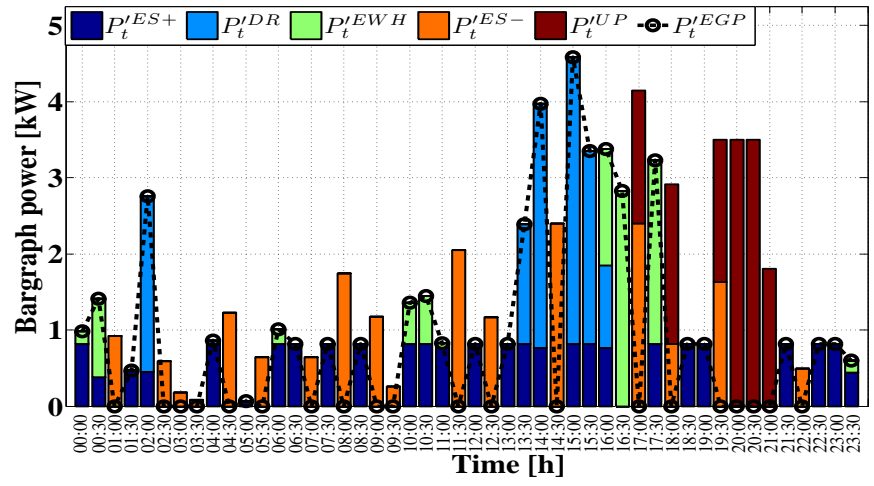

(b) EMS-PSO

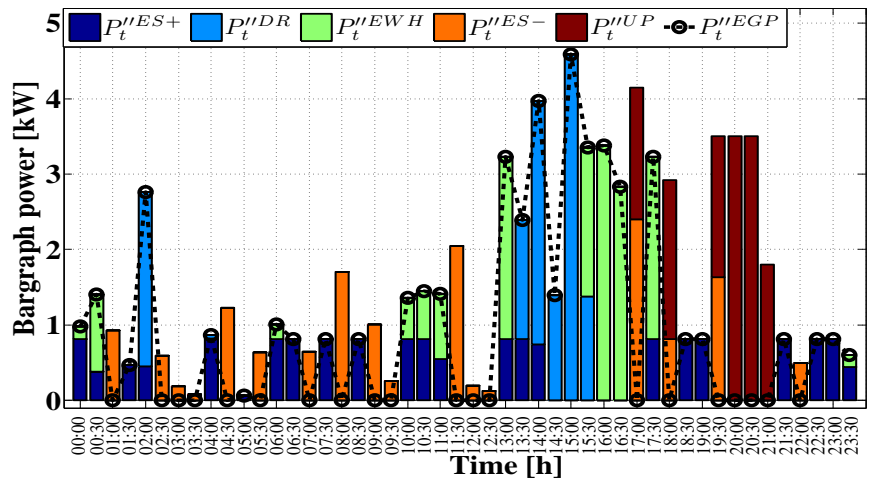

(c) EMS-MACO

Figure 8: Bar graph related to the ES power during charging and discharging, EWH, DR, UP and EGP

to $0.3 € / \mathrm{kWh}$ that is obtained at 01:00-01:30 and 04:30-05:00. In both of these hours, ES has operated in the discharging mode and has compensated shortage of power.

Furthermore, the maximum value of MCP at all algorithms is obtained during scenarios $\sharp 2$ and $\sharp 3$. As indicated in Table 3 , the average values of $\lambda_{t}^{\prime \prime M C P}$ are always less than MCP in other algorithms. Therefore, the total generation cost is reduced about 


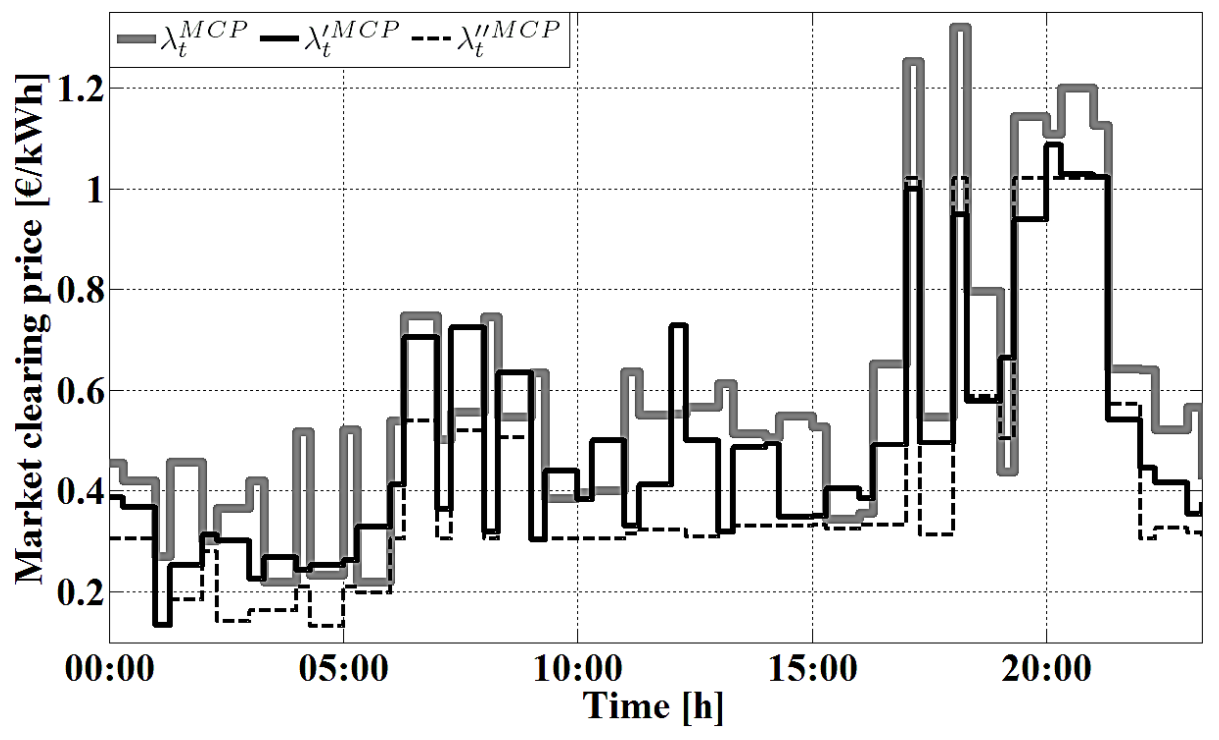

Figure 9: The value of MCP during system daily operation

Table 3: Configuration parameters for each unit

\begin{tabular}{ccccc}
\hline \hline MCP & $00: 00-06: 00$ & $06: 00-12: 00$ & $12: 00-18: 00$ & $18: 00-24: 00$ \\
\hline$\lambda_{t}^{M C P}$ & 0.62 & 0.49 & 0.56 & 0.57 \\
\hline$\lambda_{t}^{\prime M C P}$ & 0.27 & 0.43 & 0.49 & 0.52 \\
\hline$\lambda_{t}^{\prime \prime M C P}$ & 0.20 & 0.31 & 0.33 & 0.50 \\
\hline
\end{tabular}

In order to demonstrate the effectiveness and ability of the proposed algorithms in finding the optimal point with less execution time, program run times performances in both- optimization algorithms are compared. Figure 10 shows the minimization process of objective function in implemented EMSs based on PSO and MACO which occures during different iterations in optimization problems. As shown in this figure, in the best situation EMS-PSO reaches the optimal response after $90^{\text {th }}$ iteration and it is able to escape from the local peaks after $12^{\text {th }}$ iteration. Moreover, it gets close to global optimum after $65^{\text {th }}$ iteration. It can be observed that for this case the objective function converges within 22 generations. It is also noteworthy that the quality of global optimum and the convergence velocity are improved in EMS-MACO in comparison with EMS-PSO.

In order to compare the performance of the proposed algorithm with that of the other algorithms, Table 2 presents the execution time of the developed code and total generation cost of implementing all EMS-MACO and EMS-PSO algorithms. The algorithms are coded in C language and executed on a $2.53 \mathrm{GHz}$ Core(TM) Duo P8700 
personal computer with 4GB RAM. As seen in this table, run time of all proposed algorithms are less than 1 minute, but EMS-MACO has the minimum execution time. In addition, As shown in this table, the value of the objective functions in EMS-MACO is very close to EMS-MINLP [7] achieving up to a $23 \%$ reduction in execution time and around $2.3 \%$ savings on total operating costs.

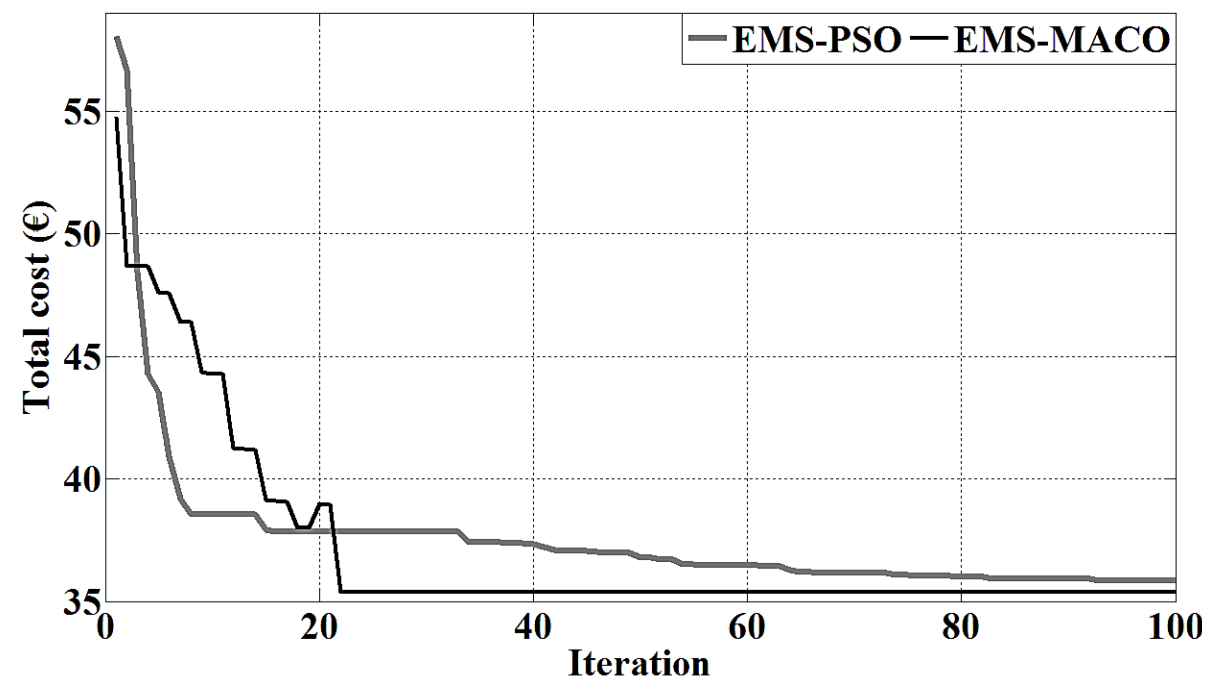

Figure 10: Improvement of objective function in two heuristic optimization algorithms

Figure 11 shows real-time scheduling output for an islanded MG by using EMSMACO algorithm for one working day with 5 minute intervals. The results show that by using the suggested algorithm, real-time scheduling is carried out. Moreover, it can ensure MG system stability under different conditions. Also, the plug and play ability has been achieved considering three mentioned scenarios. Moreover, three regions are noticable in this figure. Region A shows a situation in which WT and PV systems are out of service (due to some reasons such as fault created in the network and annual overhaul program). Under these conditions, a part of the required power is supplied by ES and MT systems. As it is observed in the figure, some of the power required by the load cannot be supplied and is shaded consequently. Region B is a typical situation at real-time performance. As it is observed, severe load fluctuations will be compensated by the systems MT and ES. At region C, scenario $\sharp 3$ has occurred. In this scenario the production of WT system reduces and EGP by PV and WT systems is at normal operation state, and the excess power is used for feeding ES, DR and/or EWH system. As observed in the figure, following this scenario the amount of power consumed by EWH is reduced.

\section{Conclusions}

A novel heuristic energy management method based on multi-layer ant colony optimization has been proposed in this paper. This algorithm uses short term forecast data (DAS, HDAS and FMRTS). Real-time scheduling has been examined to investigate the plug and play capability and the system response ability to counteract the incidents occurred in the system for load and generation variations. Then, the results obtained from EMS-MACO have been compared with other possible methods such as conventional 


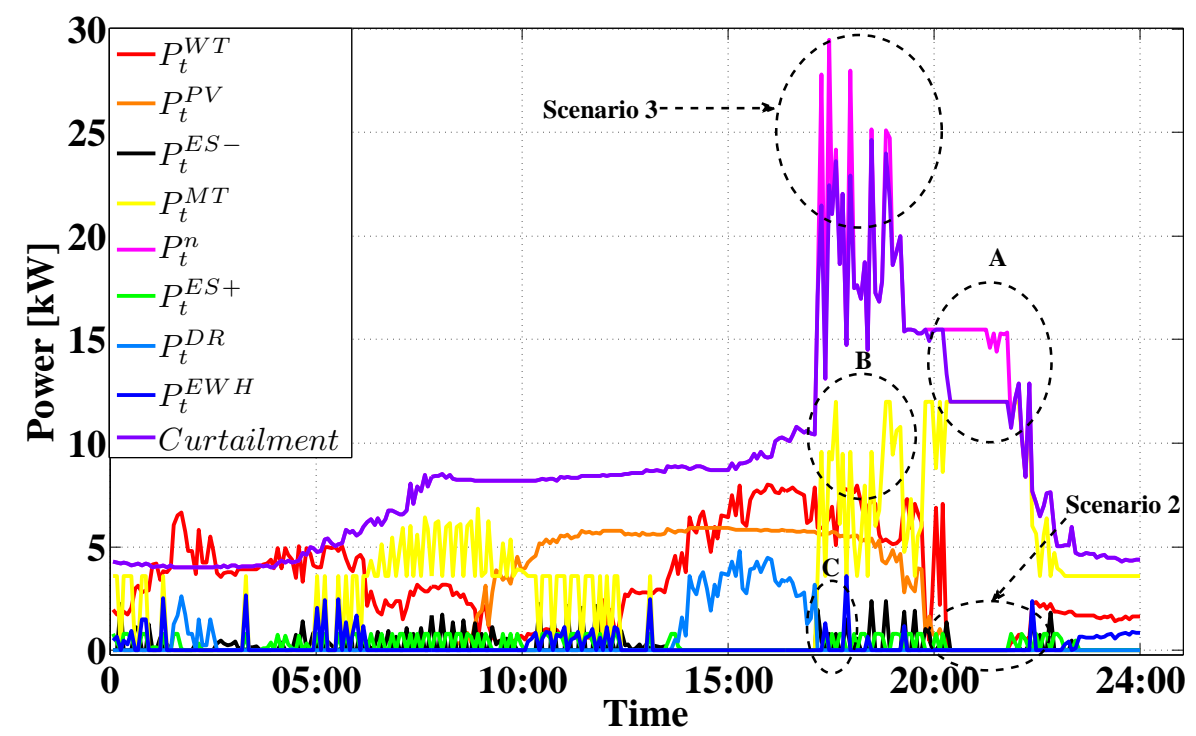

Figure 11: Real time scheduling of DERs for an islanded MG

(MCEMS) and another classical heuristic (PSO). The case study has been performed experimentally on IREC's MG by using emulators for simulating the behavior of nondispatchable energy resources and responsive/nonresponsive consumers. MACO has provided a response better than PSO algorithm due to using less iterations to converge and having less computational time. The application of MACO has reached the lowest energy cost, when compared with PSO and MCEMS. Moreover, it has ensured proper usage of the ES by reducing its cyclability as well as reducing the periods of UP. Finally, it has been demonstrated that MACO obtains closer results to the real optimal value, with the error below $1.3 \%$.

\section{Acknowledgments}

This work has been developed under the grant of European Union seventh framework program "FP7-SMARTCITIES-2013" under grant aggreement 608860.

\section{References}

[1] M. Marzband, A. Sumper, A. Ruiz-Álvarez, J. L. Domínguez-García, B. Tomoiagă, Experimental evaluation of a real time energy management system for stand-alone microgrids in day-ahead markets, Applied Energy 106 (0) (2013) $365-76$.

[2] L. Shi, Y. Luo, G. Tu, Bidding strategy of microgrid with consideration of uncertainty for participating in power market, International Journal of Electrical Power \& Energy Systems 59 (2014) 1-13.

[3] W. Liu, W. Gu, Y. Xu, S. Xue, M. Chen, B. Zhao, M. Fan, Improved average consensus algorithm based distributed cost optimization for loading shedding of 
autonomous microgrids, International Journal of Electrical Power \& Energy Systems 73 (2015) 89-96.

[4] C. W. Lou, M. C. Dong, A novel random fuzzy neural networks for tackling uncertainties of electric load forecasting, International Journal of Electrical Power \& Energy Systems 73 (2015) 34-44.

[5] E. Sfikas, Y. Katsigiannis, P. Georgilakis, Simultaneous capacity optimization of distributed generation and storage in medium voltage microgrids, International Journal of Electrical Power \& Energy Systems 67 (2015) 101-13.

[6] A. Jayawardena, L. Meegahapola, D. Robinson, S. Perera, Representation of a grid-tied microgrid as a reduced order entity for distribution system dynamics and stability studies, International Journal of Electrical Power \& Energy Systems 73 (2015) 591-600.

[7] M. Marzband, Experimental validation of optimal real-time energy management system for microgrids, $\mathrm{PhD}$ thesis, Departament d'Enginyeria Elèctrica, EU d'Enginyeria Tècnica Industrial de Barcelona, Universitat Politècnica de Catalunya (2013).

[8] M. Marzband, A. Sumper, J. L. Domínguez-García, R. Gumara-Ferret, Experimental validation of a real time energy management system for microgrids in islanded mode using a local day-ahead electricity market and MINLP, Energy Conversion and Management 76 (0) (2013) 314-22.

[9] E. Kuznetsova, C. Ruiz, Y.-F. Li, E. Zio, Analysis of robust optimization for decentralized microgrid energy management under uncertainty, International Journal of Electrical Power \& Energy Systems 64 (2015) 815-32.

[10] J. P. Fossati, A. Galarza, A. M. Villate, J. M. Echeverría, L. Fontán, Optimal scheduling of a microgrid with a fuzzy logic controlled storage system, International Journal of Electrical Power \& Energy Systems 68 (2015) 61-70.

[11] D. Merkle, M. Middendorf, H. Schmeck, Ant colony optimization for resourceconstrained project scheduling, IEEE Transactions on Evolutionary Computation 6 (4) (2002) 333-46.

[12] A. Ahuja, S. Das, A. Pahwa, An AIS-ACO hybrid approach for multi-objective distribution system reconfiguration, IEEE Transactions on Power Systems 22 (3) (2007) 1101-11.

[13] M. Dorigo, C. Blum, Ant colony optimization theory: A survey, Theoretical Computer Science, 344 (2-3) (2005) 243-78.

[14] C. Blum, Ant colony optimization: Introduction and recent trends, Physics of Life Reviews, 2 (4) (2005) 353-73.

[15] M. Dorigo, T. Stützle, Ant Colony Optimization, Ant Colony Optimization, MIT, 2004.

[16] M. Marzband, F. Azarinejadian, M. Savaghebi, J. M. Guerrero, An optimal energy management system for islanded microgrids based on multiperiod artificial bee colony combined with markov chain, IEEE systems journal, PP (99) (2015) 111 . 
[17] M. Lopez-Ibanez, T. Stutzle, The automatic design of multiobjective ant colony optimization algorithms, IEEE Transactions on Evolutionary Computation 16 (6) (2012) 861-75.

[18] J. G. Vlachogiannis, N. D. Hatziargyriou, K. Y. Lee, Ant colony system-based algorithm for constrained load flow problem, IEEE Transactions on Power Systems 20 (3) (2005) 1241-49.

[19] D. N. Simopoulos, S. D. Kavatza, C. D. Vournas, An enhanced peak shaving method for short term hydrothermal scheduling, Energy Conversion and Management, 48 (11) (2007) 3018-24.

[20] M. Marzband, A. Sumper, M. Chindriş, B. Tomoiagă, Energy management system of hybrid microgrid with energy storage, The International Word Energy System Conference (WESC), Suceava, Romania, 2012.

[21] M. Marzband, A. Sumper, Implementation of an optimal energy management within islanded microgrid, International Conference on Renewable Energies and Power Quality (ICREPQ), Cordoba, Spain, 2014.

[22] Renewable energy concepts, Available at: http : //www . renewable-energy-concepts.com/wind-energ y/wind-basics/wind-power.html.

[23] European photovoltaic solar energy conference and exhibition, Available at: http : //wWw . eupvsec-proceedings . com/, [accessed July 31 2012].

[24] Estacions automàtiques (xema), Available at: http://www.meteo.cat/xema/AppJava/SeleccioPerComarc a.do, [accessed July 31 2012].

[25] Day-ahead energy market, Available at: http://www.pjm.com/markets-and -operations/energy/day-ahead. aspx, [accessed July 31 2012]. 